\title{
HYPERSURFACES IN A CONFORMALLY FLAT SPACE WITH CURVATURE COLLINEATION
}

\author{
K. L. DUGGAL and R. SHARMA \\ Department of Mathematics and Statistics \\ University of Windsor \\ Windsor, Ontario, Canada N9B 3P4 \\ Department of Mathematics \\ University of New Haven \\ West Haven, Connecticut, 06516, U.S.A. \\ (Received February 7, 1990 and in revised form August 13, 1990)
}

\begin{abstract}
We classify the shape operators of Einstein and pseudo Einstein hypersurfaces in a conformally flat space with a symmetry called curvature collineation. We solve the fundamental problem of finding all possible forms of non-diagonalizable shape operators. A physical example of space-time with matter is presented to show that the energy condition has direct relation with the diagonalizability of shape operator.
\end{abstract}

KEY WORDS AND PHRASES: Shape operators, Proper and improper Hypersurfaces, Einstein and pseudo-Einstein hypersurfaces, curvature collineation, matter tensor.

1980 AMS SUBJECT CLASSIFICATION CODE: AMS Subject classification code: $53 C 40,53 C 50$

1. INTRODUCTION. The eigenvectors of the shape operator (second fundamental form operator) of a hypersurface of a semi-Riemannian space need not be all real. They are real for positive definite hypersurfaces. For indefinite hypersurfaces some of them may be complex and zero length (null). In the latter case the real eigenvectors (principal directions) may not span the tangent space of the hypersurface at every point. If the eigenvalues (principal normal curvatures) are real and no eigenvectors are null at every point, then the hypersurface is called proper in the terminology used by Fialkow [1]. A hypersurface which is not proper, is called improper.

Usually one prefers to embed a hypersurface in a flat (Euclidean or Minkowskian) space. But, as pointed out by Goenner [2], there is no specific reason for choosing the ambient space flat, one can consider other ambient spaces such as a space form, a Ricci-flat space, an Einstein space and a conformally flat space. Fialkow [1] provided a complete classification of proper Einstein hypersurfaces in an indefinite space form. Magid [8] has algebraically classified improper Einstein hypersurfaces in an indefinite space form. 
The aim of this paper is to present an algebraic classification of proper/improper Einstein and pseudo Einstein hypersurfaces in a conformally flat space. An example of this space-time is considered to show that the energy condition has direct relation with the diagonalizability of the shape operator.

DEFINITION 1.1. (Katzin et al [4]). A vector field $V$ in a semi-Riemannian space is said to generate a 1-parameter group of curvature collineations if it satisfies: $L_{V} R=0$, where $L_{V}$ and $R$ denote the Lie-derivative operator along $V$ and the Riemann curvature tensor, respectively.

Curvature collineation $(C C)$ is a fundamental symmetry [4] property of semi-Riemannian spaces. Indeed, it is known [4] that, for Ricci-flat spaces, more familiar symmetries such as projective and conformal collineations (including affine collineations, motions, conformal and homothetic motions) are subcases of $C C$. Physically, the well-known Komar's covariant identity [8] (which acts as a conservation law generator in general relativity) follows naturally as a necessary condition for a $C C$. Thus $C C^{\prime} s$ are necessarily embraced by the group of general curvilinear coordinate transformations.

We, therefore, choose a conformally flat embedding space $\bar{M}$ admitting a 1-parameter group of $C C^{\prime} s$. Our choice of the embedded space is a class of semi-Riemannian spaces defined as follows:

DEFINITION 1.2. A semi-Riemannian space is said to be pseudo-Einstein if there exists a 1-form $u$ such that

$$
R i c=\chi g+\eta u \otimes u
$$

and $g(U, U)=e\left(e^{2}=1\right)$; where $g(U, X)=u(X)$, Ric denotes the Ricci tensor and $\chi, \eta$ are scalar functions. For $\eta=0$, it reduces to an Einstein space.

The above definition is motivated by

(1) Yano's definition [11] of a pseudo-Einstein hypersurface of a Kaehlerian space, given by equation (1.1) with $\chi, \eta$ as constants.

(2) Einstein's field equations in the framework of general relativity [4]

$$
R i c+\left\{\wedge-\frac{1}{2} r\right\} g=p g+(\mu+p) u \otimes u,
$$

where $p$ and $\mu$ are the pressure and energy densities of a perfect fluid, $u$ is the 1 -form metrically equivalent to the velocity 4-vector $U$ and $\wedge$ stands for the cosmological constant.

2. SHAPE OPERATOR OF EINSTEIN HYPERSURFACES. First we state the following result of Katzin et al [5].

LEMMA 2.1. Let a vector field $V$ generate $C C$ in an m-dimensional conformally flat space $\bar{M}$ with metric $g$. If $\bar{M}$ is a space form then $V$ defines a motion (isometry) for $m \geq 3$ and a conformal motion for $m=2$. If $\bar{M}$ is not a space form then $L_{V} g=2 \sigma g+\tau \overline{R i c}$, where $\sigma$ and $\tau$ are scalar functions and $\overline{R i c}$ is the Ricci tensor of $\bar{M}$.

Katzin et al $[5,6]$ have shown that there are essentially only two types of conformally flat spaces admitting proper $C C$ viz. reducible and irreducible. In the reducible case $K_{1} \times K_{n-1}$ 
(scalar curvature $\neq 0$ ) the metric is

$$
d s^{2}=e_{1}\left(d x^{1}\right)^{2}+\left[1-\frac{K_{0}}{4} \sum_{2}^{n} e_{H}\left(x^{H}\right)^{2}\right]^{-2}\left[\sum_{2}^{n} e_{J}\left(d x^{J}\right)^{2}\right]
$$

where $e^{\prime} s$ are $\pm 1, K_{0}$ is the constant curvature of $K_{n-1}$ and upper case indices run from 2 to $n$. An example of such spaces is the Einstein Static Universe. Moreover, the $C C$ vector is $V^{i}=\left[V^{1}\left(x^{1}\right), V^{J}\left(x^{2}, \ldots, x^{n}\right)\right]$ where $V^{1}$ is arbitrary function of $x^{1}$ other than $a x^{1^{2}}+b x^{1}+c$ and $V^{J}$ defines a motion in $K_{n-1}$. This means that $K_{n-1}$ is a totally geodesic hypersurface of $K_{1} \times K_{n-1}$. Thus we are motivated to assume that the tangential component of the $C C$ vector in $\bar{M}$ generates a motion in $M$.

As the case when $\bar{M}$ is a space form, has been discussed in [9], we shall assume $\bar{M}$ not to be a space form in the subsequent discussions.

THEOREM 2.1. If an indefinite Einstein hypersurface $M$ of dimension $n \geq 3$, is embedded isometrically into a conformally flat space $\bar{M}$ admitting $C C$ generated by a vector field whose tangential component is Killing in $M$, then the shape operator $A$ at each point of $M$ is either diagonalizable or can be put into one of the following forms:

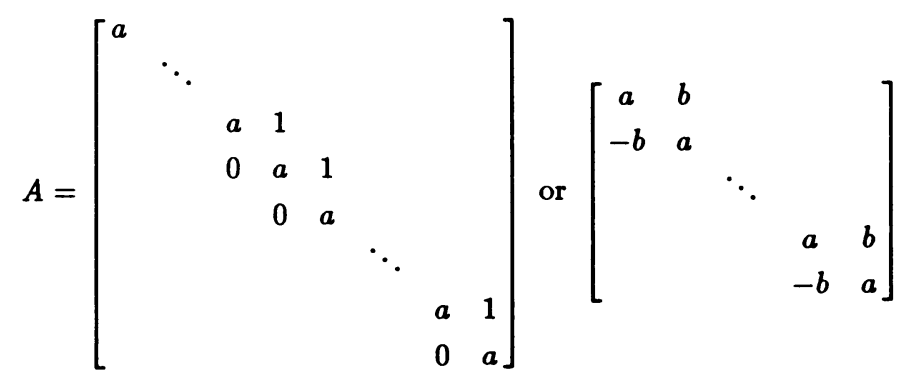

where $a$ and $b$ are arbitrary functions on $M$, with respect to some specially chosen basis. In the last case, $n$ is even and the signature of the metric of $M$ is $(n / 2, n / 2)$.

Proof: A striaghtforward application of lemma 3.1 shows

$$
L_{V} g=2 \sigma g+\tau \overline{\mathrm{Ric}}
$$

where $V$ is the vector field generating $C C$ in $\bar{M}$. We decompose $V$ into its tangential part $\xi$ and normal part $f N$ as:

$$
V=\xi+f N
$$

$N$ denoting the unit vector field $(g(N, N)=\varepsilon= \pm 1)$ normal to $M$ and $f$ a scalar function on $M$. Let us denote arbitrary vector fields tangent to $M$ by $X, Y, Z, W$. Then eqn. (2.3) obtains

$$
g\left(\bar{\nabla}_{X} V, Y\right)+g\left(\bar{\nabla}_{Y} V, X\right)=2 \sigma g(X, Y)+\tau \overline{\operatorname{Ric}}(X, Y),
$$

where $\bar{\nabla}$ denotes Levi-Civita connection of $\bar{M}$. Employing eqn. (2.4), Gauss and Weingarten formulae in the above yields

$$
\left(L_{\xi} g\right)(X, Y)=2 \sigma g(X, Y)+2 f g(A X, Y)+\tau \overline{\operatorname{Ric}}(X, Y) .
$$


As $\bar{M}$ is conformally flat, we have

$$
\begin{aligned}
g(\bar{R}(\bar{X}, \bar{Y}) \bar{Z}, \bar{W})= & {[\overline{\operatorname{Ric}}(\bar{Y}, \bar{Z}) g(\bar{X}, \bar{W})-\overline{\operatorname{Ric}}(\bar{X}, \bar{Z}) g(\bar{Y}, \bar{W})} \\
& +g(\bar{Y}, \bar{Z}) \overline{\operatorname{Ric}}(\bar{X}, \bar{W})-g(\bar{X}, \bar{Z}) \overline{\operatorname{Ric}}(\bar{Y}, \bar{W})] /(n-1) \\
& -\{\bar{r} / n(n-1)\}[g(\bar{Y}, \bar{Z}) g(\bar{X}, \bar{Z}) g(\bar{Y}, \bar{W})]
\end{aligned}
$$

The Gauss equation for $M$ is

$$
\begin{aligned}
g(\bar{R}(X, Y) Z, W)=g(R(X, Y) Z, W) & \\
& -\varepsilon\{g(A Y, Z) g(A X, W)-g(A X, Z) g(A Y, W)\}
\end{aligned}
$$

where $A$ is the shape operator of $M$. Using it in (2.6) gives

$$
\begin{aligned}
g(R(X, Y) Z, W) & =[\overline{\operatorname{Ric}}(Y, Z) g(X, W)-\overline{\operatorname{Ric}}(X, A) g(Y, W) \\
& +g(Y, Z) \overline{\operatorname{Ric}}(X, W)-g(X, Z) \overline{\operatorname{Ric}}(Y, W)] /(n-1) \\
& -\{\bar{r} / n(n-1)\}[g(Y, Z) g(X, W)-g(X, Z) g(Y, W)] \\
& +\varepsilon[g(A Y, Z) g(A X, W)-g(A X, Z) g(A Y, W)]
\end{aligned}
$$

Now, if we substitute $\bar{X}=\bar{W}=N$ and $\bar{Y}=Y, \bar{Z}=z$ in (2.6), then

$$
\begin{aligned}
g(\bar{R}(N, Y) Z, N) & =[\{\overline{\operatorname{Ric}}(N, N)-(\varepsilon \bar{r} / n)\} /(n-1)] g(Y, Z) \\
& +(\varepsilon /(n-1) \overline{\operatorname{Ric}}(Y, Z) .
\end{aligned}
$$

By the substitution $X=W=e_{i}$; where $\left\{e_{i}\right\}$ is an orthonormal frame in $M$ in eqn. (2.7), multiplying by $\varepsilon_{i}=g\left(e_{i}, e_{i}\right)$ and finally summing over $i$, we find

$$
\overline{\operatorname{Ric}}(Y, Z)-\varepsilon g(\bar{R}(N, Y) Z, N)=\overline{\operatorname{Ric}}(Y, Z)-\varepsilon\{\operatorname{tr} . A) g(A Y, Z)-g(A Y, A Z)\},
$$

keeping in mind that $\left\{e_{i}, N\right\}$ constitutes an orthonormal frame in $\bar{M}$. Eliminating $g(\bar{R}(N, Y) Z, N)$ from the last two eqns. gets

$$
\begin{aligned}
\overline{\operatorname{Ric}}(Y, Z) & =\{(n-1) /(n-2)\}[\operatorname{Ric}(Y, Z) \\
& +\{(\varepsilon n \overline{\operatorname{Ric}}(N, N)-\bar{r}) / n(n-1)\} g(y, x) \\
& +\varepsilon\{(\operatorname{tr} . A) g(A Y, Z)-g(A Y, A Z)\}]
\end{aligned}
$$

Again, substituting $Y=Z=e_{i}$, multiplying $\varepsilon_{i}$ and then summing over $i$; in the above eqn. yields

$$
\bar{r}-r=2 \varepsilon \overline{\operatorname{Ric}}(N, N)-\varepsilon\left\{(\operatorname{tr} . A)^{2}-\operatorname{tr} . A^{2}\right\} .
$$

By feeding eqn. (2.10) into (2.9) we obtain

$$
\begin{aligned}
\overline{\operatorname{Ric}}(Y, Z)=\{(n-1) /(n-2)\}[\overline{\operatorname{Ric}}(Y, Z)- & \varepsilon\{(\operatorname{tr} . A) g(A Y, Z)-g(A Y, A Z)\}] \\
& +\frac{g(X, Y)}{2(n-1)}\left[(n-2)(\bar{r} / n)-r+\varepsilon\left((\operatorname{tr} . A)^{2}-\operatorname{tr} . A^{2}\right)\right]
\end{aligned}
$$


Through eqn. (2.5) and the hypothesis that $\xi$ generates motion in $M$, we find

$$
\overline{\operatorname{Ric}}(Y, Z)=-(2 f / \tau) g(A Y, Z)+\rho g(Y, Z)
$$

where $\rho$ is a scalar function on $M$. A cumbersome computation using the last two equations shows

$$
\varepsilon Q=\phi I+\theta A-A^{2}
$$

$Q$ being defined by $g(Q X, Y)=\overline{\operatorname{Ric}}(X, Y)$, and

$$
\begin{gathered}
\varepsilon n \phi=r+\{2 f(n-2) /(n-1) \tau\} \operatorname{tr} . A-\varepsilon\left\{(\operatorname{tr} . A)^{2}-\operatorname{tr} . A^{2}\right\} \\
\theta=\operatorname{tr} . A-2 \varepsilon f(n-2) /(n-1) \tau
\end{gathered}
$$

Now, using the Petrov classification scheme [10] for symmetric tensors, $A$ can be cast into the form:

$$
A=\left[\begin{array}{cccccc}
B_{1} & & & & & \\
& \ddots & & & & \\
& & B_{k} & & & \\
& & & C_{1} & & \\
& & & & \ddots & \\
& & & & & C_{\ell}
\end{array}\right]
$$

where

$$
B_{i}=\left[\begin{array}{ccccc}
d_{i} \lambda_{i} & d_{i} & & & \\
0 & d_{i} \lambda_{i} & d_{i} & & \\
& & \ddots & & \\
& & & \ddots & d_{i} \\
& & & & d_{i} \lambda_{i}
\end{array}\right]\left(d_{i}= \pm 1\right)
$$

is an $\left(s_{i} \times s_{i}\right)$-matrix and

$$
C_{j}=\left[\begin{array}{cccccccc}
\alpha_{j} & \beta_{j} & 1 & 0 & & & & \\
-\beta_{j} & \alpha_{j} & 0 & 1 & & & & \\
& & \alpha_{j} & \beta_{j} & 1 & 0 & & \\
& & -\beta_{j} & \alpha_{j} & 0 & 1 & & \\
& & & & \ddots & & & \\
& & & & & & & \\
& & & & & \ddots & 1 & 0 \\
& & & & & & 0 & 1 \\
& & & & & & \alpha_{j} & \beta_{j} \\
& & & & & & -\beta_{j} & \alpha_{j}
\end{array}\right]\left(\beta_{j} \neq 0\right)
$$

is a $\left(2 t_{j} \times 2 t_{j}\right)$-matrix. As per our hypothesis, $M$ is Einstein, i.e. $Q=(r / n) I$. Thus, eqn. (2.11) implies that the orders of block matrices $B_{i}$ and $C_{j}$ are $\leq 2$. Consequently, $A$ has blocks of type:

$$
\left[\nu_{i}\right] \text { or }\left[\begin{array}{cc}
d_{j} \lambda_{j} & d_{j} \\
0 & d_{j} \lambda_{j}
\end{array}\right] \text { or }\left[\begin{array}{cc}
\alpha_{k} & \beta_{k} \\
-\beta_{k} & \alpha_{k}
\end{array}\right]
$$


or their combination. The second block can be transformed into $\left[\begin{array}{cc}\lambda, & 1 \\ 0 & \lambda,\end{array}\right]$ by change of the basis: $\{e, \tilde{e}\} \longrightarrow\{-e, \tilde{e}\}$. Eventually, eqn. (2.11) can be put as

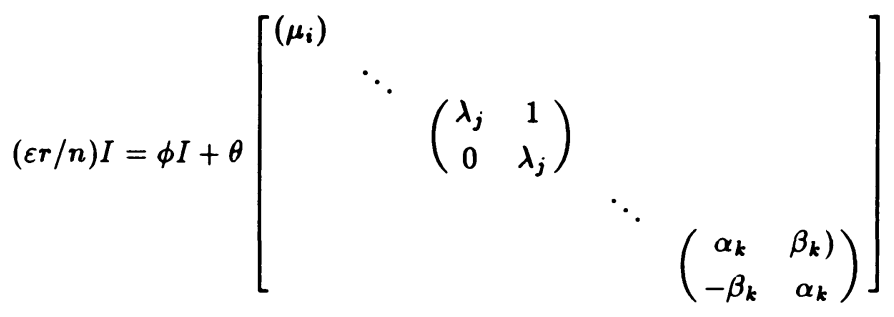

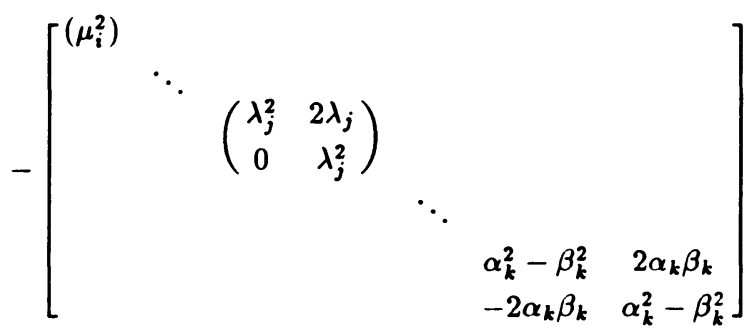

Matching corresponding entries we observe

$$
\begin{aligned}
& \theta=2 \lambda_{j}, \quad\left(\theta-2 \alpha_{k}\right) \beta_{k}=0 \\
& \theta \nu_{i}-\nu_{i}^{2}=\theta \lambda_{j}-\lambda_{j} 2=\theta \alpha_{k}-\alpha_{k}^{2}+\beta_{k}^{2}=\Phi
\end{aligned}
$$

where $\Phi=(\varepsilon r / n)-\phi$. If there are any blocks with $\beta^{\prime} s\left(\beta^{\prime} s\right.$ being non-zero) then $\alpha_{k}=\lambda_{j}=\theta / 2$, for every $j$ and $k$. Hence $\alpha^{\prime} s$ and $\lambda^{\prime} s$ are all equal to $\theta / 2$. From the last relation in (2.14) we also observe that $\beta^{\prime} s$ are all equal. Thus the relation-set (2.14) is equivalent to:

$$
\begin{aligned}
& \theta=2 \lambda, \quad \theta=2 \alpha, \\
& \nu_{i}=\left(\theta+\sqrt{\left(\theta^{2}-4 \Phi\right)}\right) / 2, \quad \lambda^{2}=\Phi, \quad \alpha^{2}+\beta^{2}=\Phi
\end{aligned}
$$

Obviously, $A$ cannot have both $\alpha$ and $\lambda$ blocks, otherwise $\lambda=\alpha$ and $\beta=0$. So, if $A$ has only $\lambda$-blocks then $\nu_{i}=\lambda$ and

$$
A=\left[\begin{array}{llllllll}
\lambda & & & & & & & \\
& \ddots & & & & & & \\
& & \lambda & 1 & & & & \\
& & 0 & \lambda & 1 & & & \\
& & & 0 & \lambda & & & \\
& & & & & \ddots & & \\
& & & & & & \lambda & 1 \\
& & & & & & 0 & \lambda
\end{array}\right], \quad \operatorname{tr} \cdot A=n \lambda
$$

From $\lambda=\theta / 2$, we get $\operatorname{tr} . A=n \theta / 2$. Thus, in virtue of eqn. $(2.15)$ we find $\theta=2 \operatorname{tr} . A=2 \lambda=$ 
$2 \varepsilon f /(n-1) \tau$. Consequently,

$$
A=\left[\begin{array}{llllllll}
a & & & & & & & \\
& \ddots & & & & & & \\
& & a & 1 & & & & \\
& & 0 & a & 1 & & & \\
& & & 0 & a & & & \\
& & & & & \ddots & & \\
& & & & & & a & 1 \\
& & & & & & 0 & a
\end{array}\right]
$$

where $a \tau(n-1)=2 \varepsilon f$. On the other hand, if $A$ has only $\alpha$-blocks then $\nu_{i}=\alpha \pm \sqrt{\left(1-\beta^{2}\right)}$ which cannot be real, because $\beta \neq 0$; and hence there cannot occur any $\nu$-term in $A$. Eventually, we find that all $\alpha^{\prime} s=\theta / 2$ and

$$
A=\left[\begin{array}{ccccc}
\alpha & \beta & & & \\
-\beta & \alpha & & & \\
& & \ddots & & \\
& & & \alpha & \beta \\
& & & -\beta & \alpha
\end{array}\right], \quad \operatorname{tr} \cdot A=n \alpha=n \theta / 2 .
$$

Thus, $\theta=2 \alpha=a$ and

$$
A=\left[\begin{array}{ccccc}
a & b & & & \\
-b & a & & & \\
& & \ddots & & \\
& & & a & b \\
& & & -b & a
\end{array}\right]
$$

This completes the proof.

COROLLARY 1. Under the hypothesis of theorem 2.1, if $M$ is improper then, either $(A-$ $a I)^{2}=0$, or $(A-a I)^{2}=-b^{2} I, a, b$ being arbitrary functions on $M$ and $I$ the identity operator.

3. SHAPE OPERATORS OF PSEUDO-EINSTEIN HYPERSURFACES. In this section we study pseudo-Einstein hypersurfaces in the same context as of the preceding section. We prove the following theorem for 4-dimensional hypersurfaces only because it is cumbersome to consider the blocks of the shape operator in higher dimensions.

THEOREM 3.1. If a 4-dimensional pseudo-Einstein hypersurface $M^{4}$ is isometrically embedded into a conformally flat space $\bar{M}^{5}$ admitting $C C$ generated by a vector field whose tangential component generates motion in $M^{4}$, then the shape operator $A$ of $M^{4}$ is either diagonalizable at each point or can be put into one of the following forms:

$$
A=\left[\begin{array}{llll}
a & & & \\
& a & & \\
& & a & 1 \\
& 0 & a
\end{array}\right] \text { or }\left[\begin{array}{cccc}
a & b & & \\
-b & a & & \\
& & a & b \\
& & -b & a
\end{array}\right] \text { or }\left[\begin{array}{cccc}
\nu \pm \sqrt{-\varepsilon e \eta} & & & \\
& \nu & & \\
& & \nu & 1 \\
& & 0 & \nu
\end{array}\right]
$$


where $a, b, \nu$ and $\eta$ are scalar function on $M^{4}$ and $e^{2}=\varepsilon^{2}=1$, with respect to some specially chosen basis.

Proof: With respect to an orthonormal basis formed by $U$ and three orthonormal vectors orthogonal to $U$, we observe that the Ricci map can be represented by

$$
Q=\left[\begin{array}{cccc}
x+e \eta & & & \\
& x & & \\
& & x & \\
& & & x
\end{array}\right]
$$

where $e=g(U, U)= \pm 1$. Proceeding exactly as in the proof of theorem 2.1, up to eqn. (2.11) we find that $A$ has blocks of type

$$
\left[\nu_{i}\right] \text { or }\left[\begin{array}{cc}
\lambda_{j} & 1 \\
0 & \lambda_{j}
\end{array}\right] \text { or }\left[\begin{array}{cc}
\alpha_{k} & \beta_{k} \\
-\beta_{k} & \alpha_{k}
\end{array}\right]
$$

Therefore, either $A$ will be diagonalizable at each point or can be put into one of the following forms:

$$
\begin{aligned}
& \text { (1) }\left[\begin{array}{llll}
\nu_{i} & & & \\
& \nu_{2} & & \\
& & \lambda & 1 \\
& & 0 & \lambda
\end{array}\right] \\
& \text { (3) }\left[\begin{array}{cccc}
\lambda_{1} & 1 & & \\
0 & \lambda_{1} & & \\
& & \lambda_{2} & 1 \\
& & 0 & \lambda_{2}
\end{array}\right] \\
& {\left[\begin{array}{cccc}
\nu_{1} & & & \\
& \nu_{2} & & \\
& & \alpha & \beta \\
& & -\beta & \alpha
\end{array}\right]} \\
& {\left[\begin{array}{cccc}
\alpha_{1} & \beta_{1} & & \\
-\beta_{1} & \alpha_{1} & & \\
& & \alpha_{2} & \beta_{2} \\
& & -\beta_{2} & \alpha_{2}
\end{array}\right]}
\end{aligned}
$$

Plugging the above listed forms of $A$ into eqn. (2.11) shows that the type (2) is not possible whereas the types (3) and (4) hold only if $M^{4}$ is Einstein which is taken care of by theorem 2.1. The remaining type (1) leads to:

$$
\nu_{2}=\lambda=\theta / 2, \quad \nu_{1}=\nu_{2}+\sqrt{(-e \varepsilon \eta)}
$$

Thus, in this case

$$
A=\left[\begin{array}{llll}
\nu \pm \sqrt{(-e \varepsilon \nu)} & & & \\
& \nu & & \\
& & \nu & 1 \\
& & 0 & \nu
\end{array}\right]
$$

Where $\nu=\theta / 2$ and $\operatorname{tr} . A=(8 \varepsilon f / 3 \tau) \mp \sqrt{(-e \varepsilon \eta)}$.

COROLLARY 2.. Under the hypothesis of theorem 3.1, if $M^{4}$ is not Einstein then $A$ is either diagonalizable at each point or can be put in the form

$$
A=\left[\begin{array}{llll}
\nu \pm \sqrt{(-e \varepsilon \eta)} & & & \\
& \nu & & \\
& & \nu & 1 \\
& & 0 & \nu
\end{array}\right]
$$


with respect to some specially chosen basis.

We now interpret the physical significance of a pseudo-Einstein space in the light of the above corollary. Einstein's field equations of general relativity can be written in suitable units as:

$$
\operatorname{Ric}+\left(\wedge-\frac{1}{2} r\right) g=T
$$

where $T$ is the energy-momentum tensor of matter distribution. A comparison of eqn.(3.1) with the defining eqn.(1.1) of a pseudo Einstein space, shows that

$$
T=p g+(\mu+p) u \otimes u
$$

where $p$ and $\mu$ are given by

$$
p+\frac{1}{2} r-\wedge=\chi, \quad \mu+p=\eta
$$

Eqn. (3.2) represents an isotropic matter of type I [3] with energy density $\mu$ and pressure $p$, provided $g(U, U)=-1$ (signature of $M^{4}$ being -+++ ). It is remarkable to observe that such a space is non-Einstein because otherwise we would get the non-physical state $\mu+p=0$.

The signature of $\bar{M}^{5}$ could be -++++ or --+++ only. If $M^{5}$ has the former (Lorentzian) signature then $\varepsilon=1$. Moreover, since $g(U, U)=-1$ we have $e=-1$. Hence $\sqrt{(-e \varepsilon \eta)}=\sqrt{(\mu+p)}$ which is always non-zero real (because $\mu+p>0$ ). Thus, in this case either $A$ is diagonalizable at each point or

$$
A=\left[\begin{array}{llll}
\nu \pm \sqrt{(\mu+p)} & & & \\
& \nu & & \\
& & \nu & 1 \\
& & 0 & \nu
\end{array}\right]
$$

On the other hand, if the signature of $\bar{M}^{5}$ is --+++ , then we have $\varepsilon=e=-1, \sqrt{(-\varepsilon e)}=$ $\sqrt{\{-(\mu+p)\}}$ which is always imaginary for physically realistic matter. Consequently, $M$ is proper. For this later case, the energy condition: $\mu+p>0$, is equivalent to saying that $M^{4}$ is proper.

4. EXAMPLE. Isometric embeddings of space-times has been used to obtain deeper insight into the geometrical properties of the embedded space-time. This technique has also shed some light on a number of global questions concerning singularities and causality properties. In particular, several physically useful exact solutions have been found by the embedding technique, at least for some cases of low embedding class [the maximum number of extra dimensions is called the embedding class]. In fact, the maximal analytic extension of the Schwarzschild solution [3] was found by the method of embedding.

In support of the above, we present an example of an isometric embedding of the type of space-time described in this paper viz., 4-dimensional space-time of general relativity with an isotropic matter of type I [3]. For details, we refer [7].

A 4-dimensional space-time is of embedding class 1 (i.e., a hypersurface) if there exists a symmetric tensor $\Omega_{a b}$ satisfying:

$$
\begin{aligned}
R_{a b c d} & =e\left(\Omega_{a c} \Omega_{b d}-\Omega_{a d} \Omega_{b c}\right), \quad e= \pm 1 \quad \text { (Gauss) } \\
\Omega_{a b ; c} & =\Omega_{a c ; b} \quad(\text { Codazzi) }
\end{aligned}
$$


The field equations then yield (for $\Lambda=0$ ).

$$
R_{a b}=T_{a b}+\frac{1}{2} r g_{a b}=e\left(\Omega_{a b} \Omega_{c}^{c}-\Omega_{a c} \Omega_{b}^{c}\right)
$$

All possible tensors $\Omega$ corresponding to the isotropic matter or Maxwell type are known. Precisely, there are four different cases (for details, see [7], pages 360-367). Here we mention the following one case related to this paper:

ISOTROPIC PETROV TYPE 0 SOLUTION.

$$
\begin{aligned}
& T=(\mu+p) u \otimes u+p g, \quad \Omega=A u \otimes u+B g, \\
& \mu+p=2 A B>0, \quad \mu=3 B^{2}>0, \quad e=1
\end{aligned}
$$

Relating this case to the equations (3.2) and (3.3) we get

$$
\eta=2 A B, \quad \chi=2 A B-3 B^{2}+\frac{1}{2} r \text { and } \Lambda=0
$$

\section{REFERENCES}

1. A. Fialkow, Hypersurfaces of a space of constant curvature, Ann. of Math. 39 (1938), 762-785.

2. 'H.F. Goenner, Local isometric embedding of Riemannian manifolds and Einstein's theory of gravitation, "General Relativity and Gravitation I (one hundred years after the birth of Albert Einstein)," ed. A. Held, 1980, pp. 441-468.

3. S.W. Hawking and G.F.R. Ellis, The large scale structure of space-time, "Cambridge University Press," Cambridge, 1964.

4. G.H. Katzin, J. Levine and W.R. Davis, Curvature collineations: A fundamental symmetry property of the space-times of general relativity defined by the vanishing Lie-derivative of the Riemann curvature tensor, $\mathrm{J}$. Math. Phys. 10 (1969), 617-629.

5. G.H. Katzin, J. Levine and W.R. Davis, Curvature collineations in conformally flat spaces I, Tensor, N.S. 21 (1970), 51-61.

6. G.H. Katzin, J. Levine and W.R. Davis, Curvature collineations in conformally flat spaces II, Tensor, N.S. 21 (1970), 319-329.

7. D. Kramer, H. Stephani, M. MacCallum and E. Herlt, Exact solutions of Einstein's Field Equations, "Cambridge University Press," Cambridge, 1980.

8. A. Komar, Covariant conservation laws in general relativity, Phys. Rev. 113 (1959), 934-936.

9. M.A. Magid, Shape operators of Einstein hypersurfaces in indefinite space forms, Proc. Amer. Math. Soc. 84 (1983), 237-242.

10. A.Z. Petrov, Einstein spaces, "Pergamon Press," Oxford, 1969.

11. K. Yano and M. Kon, CR submanifolds of Kaehlerian and Sasakian manifolds, Birkhäuser, Boston. 


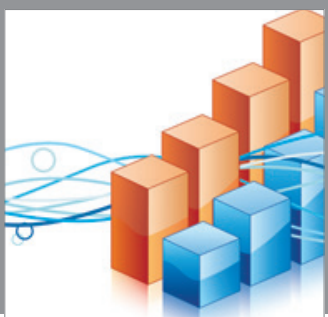

Advances in

Operations Research

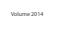

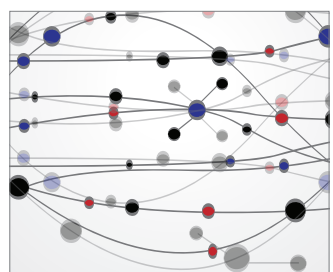

\section{The Scientific} World Journal
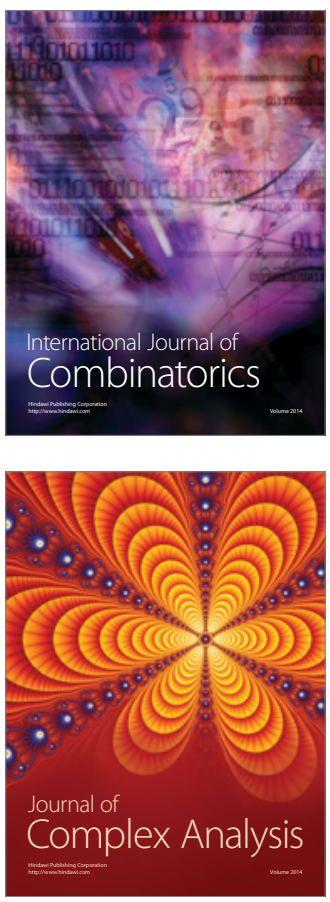

International Journal of

Mathematics and

Mathematical

Sciences
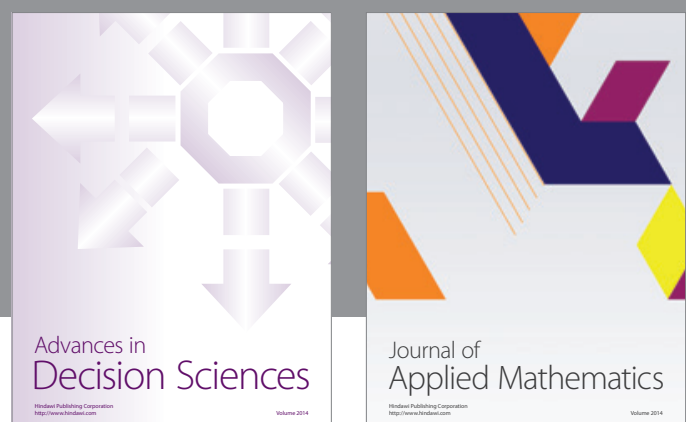

Journal of

Applied Mathematics
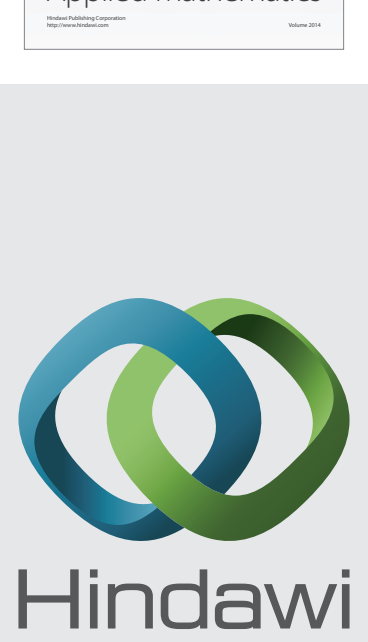

Submit your manuscripts at http://www.hindawi.com
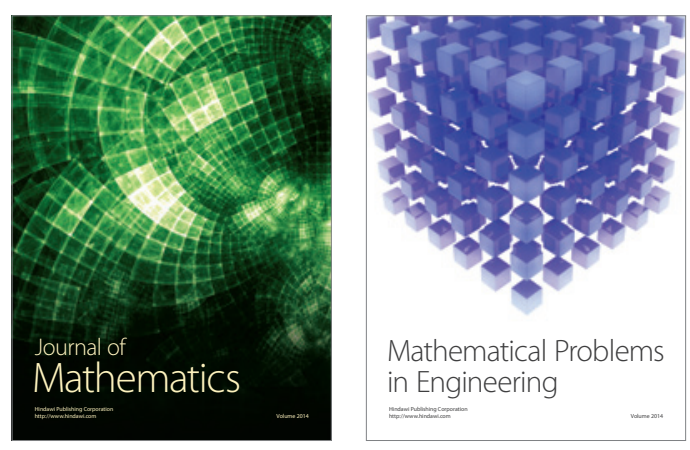

Mathematical Problems in Engineering
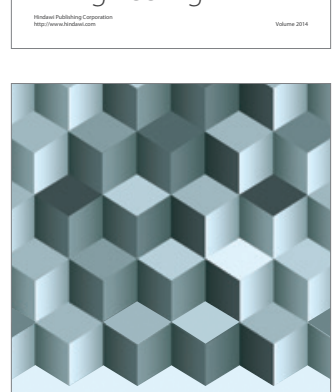

Journal of

Function Spaces
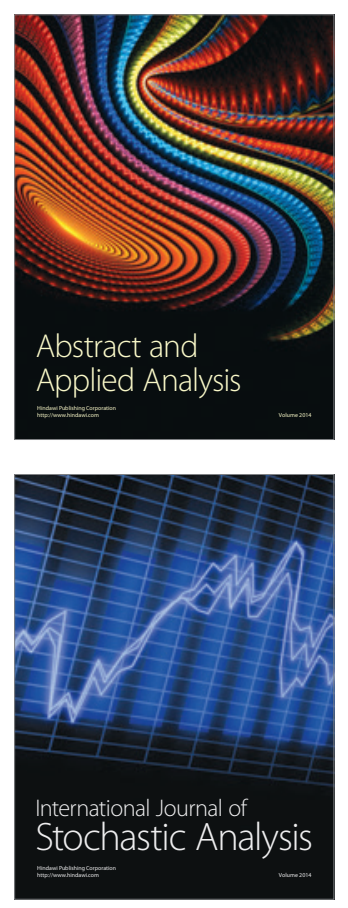

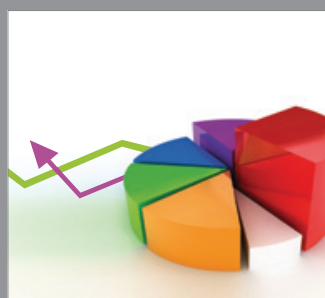

ournal of

Probability and Statistics

Promensencen
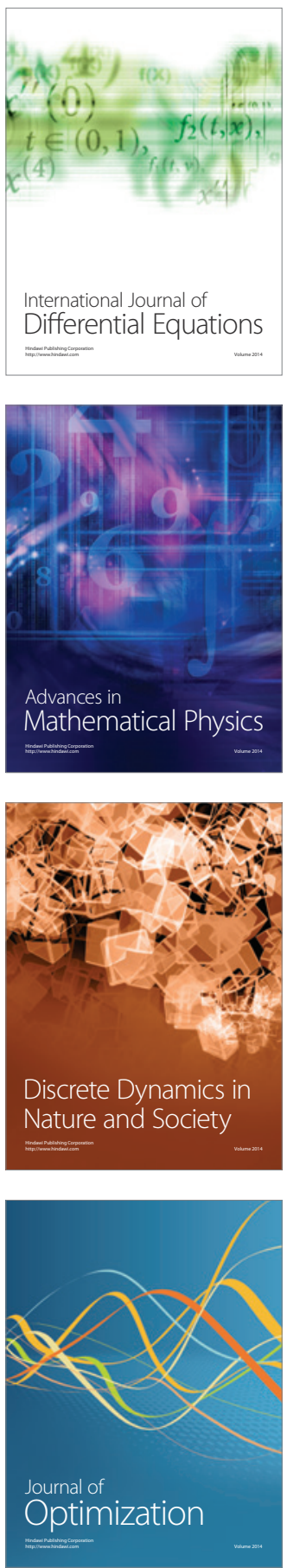